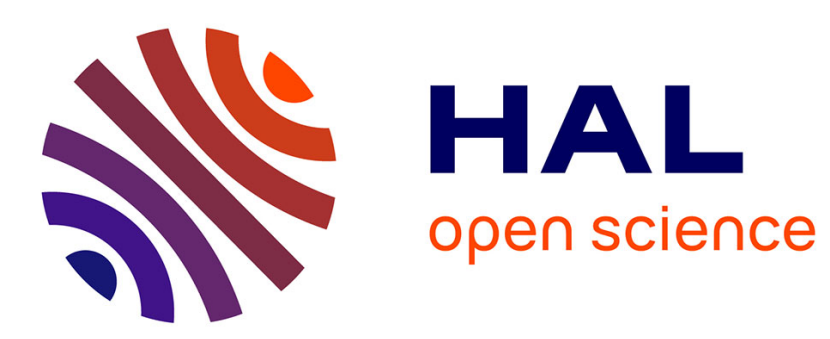

\title{
Turbo-FSK, a physical layer for LPWA: Synchronization and Channel estimation
}

Francois Dehmas, Valérian Mannoni, Vincent Berg

\section{To cite this version:}

Francois Dehmas, Valérian Mannoni, Vincent Berg. Turbo-FSK, a physical layer for LPWA: Synchronization and Channel estimation. EUCNC 2018 - European Conference on Networks and Communications, Jun 2018, Ljubljana, Slovenia. cea-01846011

\section{HAL Id: cea-01846011 https://hal-cea.archives-ouvertes.fr/cea-01846011}

Submitted on 20 Jul 2018

HAL is a multi-disciplinary open access archive for the deposit and dissemination of scientific research documents, whether they are published or not. The documents may come from teaching and research institutions in France or abroad, or from public or private research centers.
L'archive ouverte pluridisciplinaire HAL, est destinée au dépôt et à la diffusion de documents scientifiques de niveau recherche, publiés ou non, émanant des établissements d'enseignement et de recherche français ou étrangers, des laboratoires publics ou privés. 


\title{
Turbo-FSK, a physical layer for LPWA: Synchronization and Channel estimation
}

\author{
François Dehmas, Valérian Mannoni and Vincent Berg \\ CEA, LETI, MINATEC Campus, \\ F-38054 Grenoble, France \\ \{francois.dehmas, valerian.mannoni, vincent.berg\}@cea.fr
}

\begin{abstract}
Turbo Frequency Shift Keying (Turbo-FSK) has been considered as a promising physical layer for low power wide area applications. Its constant envelope at the transmitter combined with performance close to the Shannon's limit enable to achieve a high energy efficiency. However, results published so far in the literature for this waveform have assumed perfect synchronization and channel estimation. This paper, presents a synchronization and channel estimation approach based on a specifically built preamble and adapted to the performance of the new modulation. Simulations have been performed for both time and frequency synchronization as well as channel estimation. Less than $1 \mathrm{~dB}$ degradation in comparison to perfect detection is achieved for the most severe types of channels.
\end{abstract}

Keywords-LPWA; OFDM; Turbo Codes; FSK; Zadoff-Chu; Constant Envelope

\section{INTRODUCTION}

Machine type communications (M2M) are rapidly expanding such that more than twenty billion devices are expected to be connected through wireless systems by 2020 [1]. Low Power Wide Area (LPWA) network technologies constitutes an important part of the Internet of Things (IoT) by providing a long range and low power wireless connectivity alternative to current generations of cellular systems $(2 \mathrm{G}, 3 \mathrm{G}$ and $4 \mathrm{G})[2]$. LPWA is a generic term for a group of technologies that enable wide area communications at low cost and long battery life (Sigfox, LoRa, RPMA, NB-IoT, Weightless-P, IEEE $802.11 \mathrm{ah}$ ) [2]. Among them, LoRa and NB-IoT are two leading emergent technologies [3]. When 5G IoT connectivity is considered, LPWA is predicted to represent approximately $10 \%$ of the overall IoT connections [4].

For these systems, long range is achieved by ensuring a very low level of sensitivity at the receiver obtained by ensuring Quality of Service (QoS) for poor levels of Signal-to-Noise Ratio (SNR). The low energy consumption requirement directly results from the necessity to have a long battery life. At the physical layer, this is achieved by selecting an energy efficient modulation combined with an efficient usage of the Power Amplifier (PA), the most power-consuming component of the transmission chain [5]. The efficiency of the PA is highly dependent on the peak power of the signal [6]. Constant envelope modulations are thus of prime importance, as they allow for large PA efficiency gains.

Turbo Frequency Shift Keying (Turbo-FSK) is a new waveform introduced in [7] that combines an orthogonal modulation with a convolutional code. Its receiver relies on turbo processing to meet a performance close to Shannon's limit for low spectral efficiency. Furthermore, the waveform exhibits a constant envelope (i.e. it has a peak to average power ratio (PAPR) equal to $0 \mathrm{~dB}$ ). Turbo-FSK is therefore well adapted to future LPWA systems notably for the $5^{\text {th }}$ generation of cellular systems.

As Turbo-FSK proposes to improve energy efficiency to within a few dB of Shannon's limit, expected signal-to-noise ratio at the receiver should be well below $0 \mathrm{~dB}$. This imposes significant performance constraints on both synchronization (time and frequency) and channel estimation at the receiver. However, results published so far in the literature for the waveform [7][8] have assumed perfect synchronization and channel estimation. Hence, a new approach of synchronization and channel estimation based on a preamble and adapted to the performance of the new modulation is here proposed and evaluated.

The paper is structured as follows: Section II presents the principles of Turbo-FSK. In Section III, a structure of preamble adapted to the new waveform is presented. Synchronization and channel estimation algorithms are described and individually evaluated. Performance comparison to perfect synchronization and channel estimation is given in Section IV. Section V concludes the paper.

\section{PRINCIPLES OF TURBO-FSK}

In this section, a brief introduction of the Turbo-FSK scheme presented in [7] is given. The structure of the transmitter (Fig. 1) is composed of $\lambda$ stages, each one encoding a differently interleaved version of the $Q$ input information bits. At each stage information bits are grouped into $N_{q}$ blocks of $q$ bits $(Q=$ $N_{q} q$ ), and each block is sequentially encoded using a parity accumulator. The resulting $q+1$ bits of each block are then mapped to a complex codeword of the so called coplanar FSK alphabet [8] (i.e. mapped to $N_{L}$-PSK subsets of $N_{\perp}$-FSK possible symbols, $\left.q+1=\log _{2}\left(N_{L} N_{\perp}\right)\right)$. Thanks to the accumulator, every consecutive Coplanar FSK symbol is related to the previous ones. The output of each encoder is then a set of coplanar FSK codewords. A Parallel-to-Serial Conversion follows to transmit the FSK codewords through the channel.

The receiver, depicted in Fig. 2, includes a Serial-to-Parallel Conversion, to reconstruct the emitted $\lambda$ stages. A soft FSK detector estimates the probabilities of each possible codeword. 


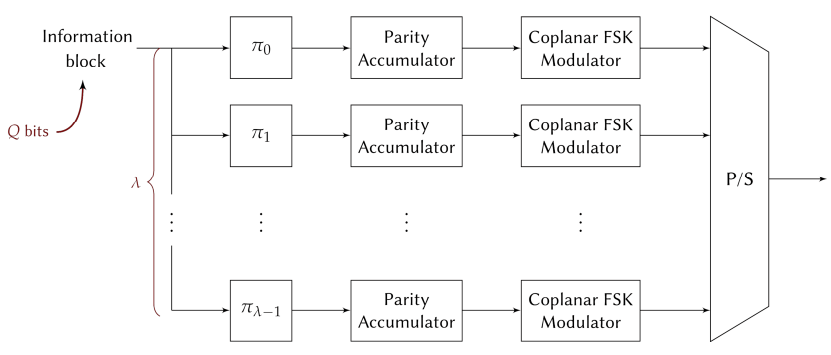

Fig. 1. Architecture of the Turbo-FSK transmitter

Then these probabilities feed the decoder, which uses them as channel observation, while output of the other decoders will be used as a priori information. A modified BCJR algorithm [9] is used to decode the trellis, and the A Posteriori Probabilities (APP) of the information bits are computed. For detailed explanations about the receiver's computations, the reader is invited to refer to [8].

Asymptotic performance for perfect interleaving can give near error free performance for an energy per information bit over the spectral density of noise $\left(E_{b} / N_{0}\right)$ of less than $0.3 \mathrm{~dB}$ and $1.35 \mathrm{~dB}$ with block size $Q$ limited to 1000 bits when assuming perfect synchronization and channel estimation [8].

Digital baseband front-end can be implemented similarly to Orthogonal Frequency Division Multiplexing (OFDM) systems using fast Fourier transform (FFT) and inverse FFT (IFFT) respectively at the receiver and the transmitter [8]. The size of the transform is denoted $N$ and the frequencies spacing, which is also the symbol rate before cyclic prefix addition, is $\Delta f$. Thus the sampling rate is $N \Delta f$.

\section{SYNCHRONIZATION AND CHANNEL ESTIMATION}

\section{A. Preamble based dectection}

A preamble-based synchronization has been considered. The preamble shall allow to get time and frequency synchronization while tolerating carrier frequency offset (CFO). In order to keep the low-power advantage of the waveform we imposed for the preamble to have a constant envelope. As a consequence, constant amplitude, zero autocorrelation (CAZAC) sequences have been considered as these are well adapted. Zadoff-Chu (ZC) sequences [10] are an example of such sequences and have already been widely used in cellular systems [11].

A preamble adapted to Turbo-FSK is thus proposed. It is composed of a ZC sequence $\boldsymbol{s}=\left(s_{0}, s_{1}, \ldots, s_{N_{p^{-1}}}\right)$ repeated $N_{a}$ times and weighted by another constant amplitude sequence

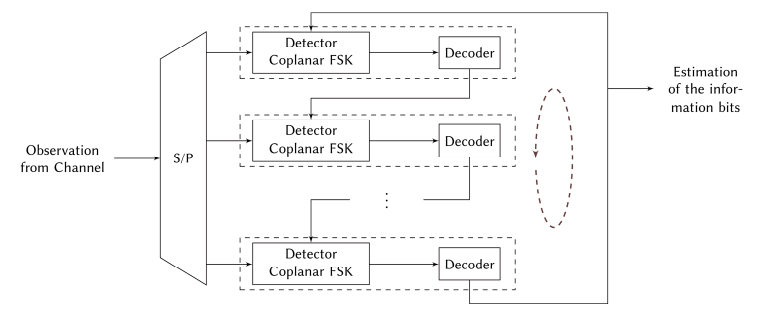

Fig. 2. Architecture of the Turbo-FSK receiver. $\boldsymbol{w}=\left(w_{0}, w_{1}, \ldots, w_{N_{a}-1}\right)$ such that the complete preamble is equal to $\boldsymbol{w} \otimes \boldsymbol{s}$, where $\otimes$ is the Kronecker vector product (Fig. 3).

The sequence $\boldsymbol{s}$ is defined as a modified ZC sequence whose phase is scaled to fit the number of frequencies $N_{\perp}$ and spectrally translated to fit the spectrum location of the FSK waveform:

$$
s_{n}=A \exp \left(-j \frac{\pi n^{2} N_{\perp}}{N N_{p}}\right) \times \exp \left(j \frac{2 \pi f_{h} n}{N \Delta f}\right)
$$

where $A$ is a constant amplitude scaling, $f_{h}$ is the upper frequency of the FSK baseband signal, $n \in \llbracket 0, N_{p}-1 \rrbracket$.

Let define $\boldsymbol{w}$ as the cumulative product of a ZC sequence:

$$
w_{n}=\prod_{i=0}^{n} \exp \left(-j \frac{\pi i^{2}}{N_{a}}\right)
$$

for $n \in \llbracket 0, N_{a}-1 \rrbracket$

To the contrary of the aforementioned cellular systems [11] whose synchronization sequences are transmitted in the frequency domain, the resulting preamble $\boldsymbol{w} \otimes \boldsymbol{s}$ has a constant envelope. It exhibits properties that are exploited in the next sections of this paper.

\section{B. Turbo-FSK Synchronization}

As Turbo-FSK should provide quality of service for low levels of SNR, preamble synchronization has to be performed using a cross-correlation between the received signal and a relatively long sequence in order to extract signal information from noise. However, in presence of significant amount of CFO (i.e. $\mathrm{CFO}>10 \mathrm{ppm}$ ) the cross-correlation peak is significantly degraded.

Instead, we suggested to cross-correlate the received signal with the shorter sequence $\boldsymbol{s}$. The result of this cross-correlation is then differentially cross-correlated to the $\boldsymbol{w}$ sequence.

More explicitly, if $r[k]$ is the received signal at instant $k$, cross-correlation with sequence $\boldsymbol{s}$ is calculated for every instant $k$ :

$$
c[k]=\sum_{n=0}^{N_{p}-1} s_{n}^{*} r[k+n]
$$

where $(\cdot)^{*}$ stands for complex conjugate.

Differential cross-correlation on the resulting signal is then equal to:

$$
d[k]=\sum_{n=0}^{N_{a}-2} w_{n+1} w_{n}^{*} c\left[k+(n+1) N_{p}\right] c^{*}\left[k+n N_{p}\right]
$$

By construction of $\boldsymbol{w}$, the sequence $\left(w_{n+1} w_{n}^{*}\right)_{0 \leq n<N_{a}-1}$ is also a $Z C$ sequence but truncated by its last element. Detecting the peak in $d[k]$ at position $k_{0}$ gives the synchronization time at the receiver. Using $N_{\perp}=32, N_{a}=128, N_{p}=32, N=128$, $\Delta f=15 \mathrm{kHz}$ Fig. 4 gives an example of a resulting $d[k]$ signal (without any noise) where we can observe a unique cross- 


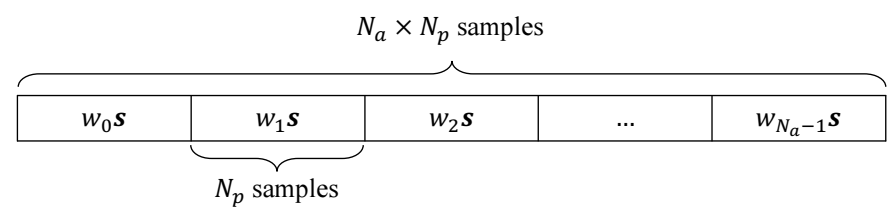

Fig. 3. Preamble structure

correlation peak that is more than $30 \mathrm{~dB}$ above the rest of the cross-correlation lags. As a unique peak is present, no start of frame delimiter is required to detect the beginning of the payload thus reducing the signaling overhead.

CFO estimation is evaluated on the samples $c\left[k_{0}\right], c\left[k_{0}+\right.$ $\left.N_{p}\right], \ldots, c\left[k_{0}+\left(N_{a}-1\right) N_{p}\right]$ using the Mengali estimator [12].

This synchronization algorithm has then been simulated and performance in terms of Packet Error Rate (PER) has been measured (assuming perfect channel estimation) in order to evaluate the performance loss due to the synchronization (for both time and frequency errors) on the receiver. The results are given in Fig. 5. The following assumptions have been taken for the simulations: $N_{\perp}=32, N_{L}=16, \lambda=4, Q=1008$ information bits, $\Delta f=15 \mathrm{kHz}$, additive white Gaussian noise (AWGN) channel. $N_{p}$ is chosen equal to 32 to support a $\mathrm{CFO} \sim$ $15 \mathrm{kHz}$ (16 ppm when in 868 or $915 \mathrm{MHz}$ ISM bands).

Using these assumptions, performance loss introduced by the synchronization algorithm is less than $0.1 \mathrm{~dB}$ when $N_{a}$ is equal to 128 at PER $=1 \%$. With a lower value $\left(N_{a}=64\right)$, the loss is $1.4 \mathrm{~dB}$ due to a lower correlation peak causing more miss detections.

In Fig. 6, the accuracy of the CFO estimation has been given using the remaining error in CFO of the $99 \%$ quantile as a function of $E_{b} / N_{0}$ for an initial frequency error of respectively $0 \mathrm{~Hz}$ and $15 \mathrm{kHz}$. In both cases, the remaining error is the same, about $50 \mathrm{~Hz}$ for an $E_{b} / N_{0} \approx 1 \mathrm{~dB}$ meaning that phase rotation is still superior to $2 \pi$ with a typical frame length of 1008 bits. This will be compensated thanks to channel estimation and further described in section IV.

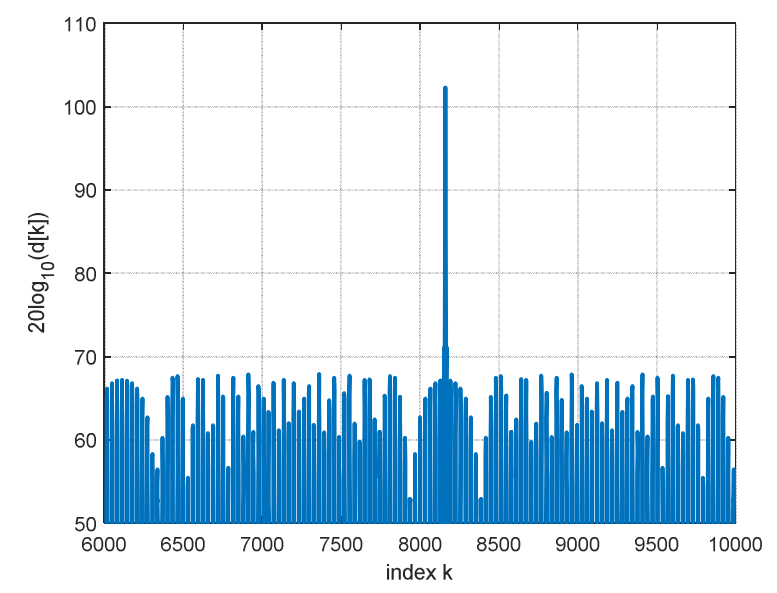

Fig. 4. Correlation on differential signal $d[k]$.

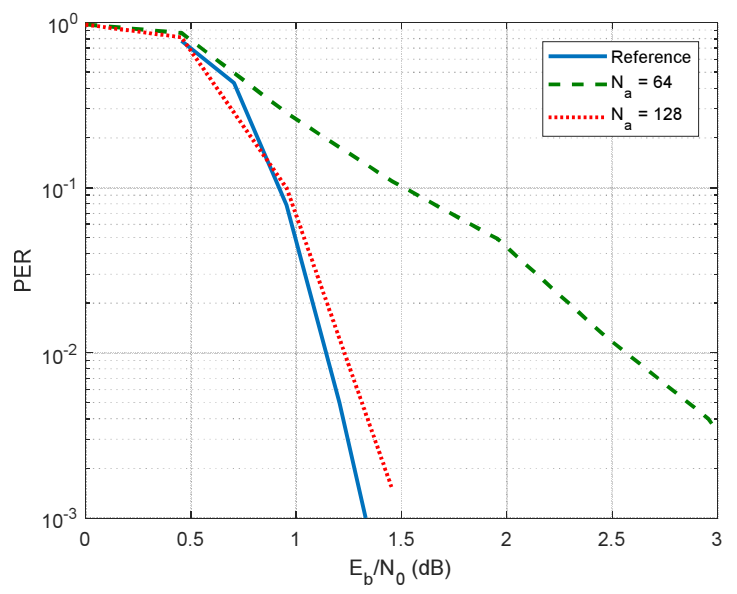

Fig. 5. Paquet Error Rate (PER) with synchronization for different preamble lengths, AWGN channel, perfect synchronization is referred as Reference.

\section{Turbo-FSK channel estimation}

Channel estimation is another mandatory operation at the Turbo-FSK receiver since coherent reception is required for the linear modulation detection (phase estimation are necessary) and for log-likelihood ratio estimations required for the BCJR algorithm [8]. The channel is assumed constant over the duration of a packet (a few 10s of ms). As the demodulation is performed in the frequency domain as is often the case in OFDM, the widespread frequency domain channel estimation approach has been considered.

Pilots for channel estimation have also been constrained to have a constant envelope. As for time and frequency synchronization, a modified time domain ZC sequence is considered to guarantee constant amplitude in the time domain and a good repartition of the power in the frequency domain. The following channel estimation sequence has been considered:

$$
p_{n}=A \exp \left(-j \frac{\pi n^{2} N_{\perp}}{N^{2}}\right) \times \exp \left(\frac{j 2 \pi f_{h} n}{N \Delta f}\right)
$$

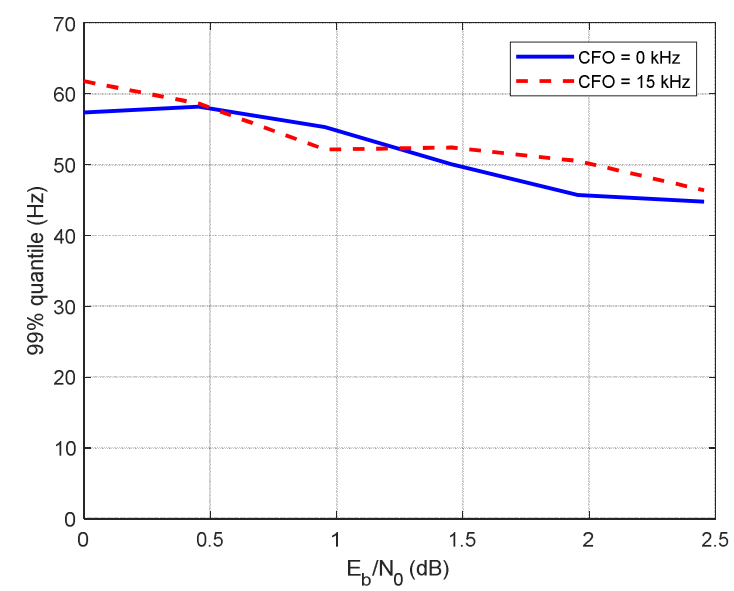

Fig. 6. CFO estimation accuracy. 
If $\boldsymbol{P}=\left(P_{0}, \ldots, P_{N-1}\right)^{T}$ is the discreet Fourier transform (DFT) of $p_{n}$, we get:

$$
R_{k}=H_{k} P_{k}+B_{k}
$$

where $\boldsymbol{H}=\left(H_{0}, \ldots, H_{N-1}\right)^{T}$ is the DFT of the channel impulse response and $\boldsymbol{B}=\left(B_{0}, \ldots, B_{N-1}\right)$ the DFT of the complex additive white Gaussian noise with a variance $\sigma^{2}$.

Different channel estimators have been compared. The least square (LS) estimator:

$$
\widehat{\boldsymbol{H}}_{\boldsymbol{L S}}=\boldsymbol{R} \operatorname{diag}(\boldsymbol{P})^{-1}
$$

where $\operatorname{diag}(\boldsymbol{P})$ stands for diagonal matrix with the vector $\boldsymbol{P}$ on its diagonal. [14]:

The linear minimum mean square error estimator (LMMSE)

$$
\widehat{\boldsymbol{H}}_{\boldsymbol{L M} \boldsymbol{M S E}}=\boldsymbol{R}_{\boldsymbol{H}}\left(\boldsymbol{R}_{\boldsymbol{H}}+\sigma^{2}\left(\operatorname{diag}(\boldsymbol{P}) \operatorname{diag}(\boldsymbol{P})^{H}\right)^{-1}\right)^{-1} \widehat{\boldsymbol{H}}_{\boldsymbol{L S}}
$$

where $\boldsymbol{R}_{\boldsymbol{H}}$ is the covariance matrix of the DFT of the channel.

An approximation of the LMMSE estimator avoiding noise power and covariance matrix estimation, can be considered supposing a uniform channel and a given (target) SNR [15].

A pragmatic improvement of LS estimator (called LS filtered in the following) has also been considered. In this case, the LS estimate is windowed in the time domain to retain only the most significant taps of the channel impulse response and filter out the noise on the channel estimate. This is equivalent to filter the frequency response of the channel to reduce the noise level and take into account the correlation between neighboring frequency taps.

Using an extended typical urban (ETU) channel [11], we compared the root mean square error (RMSE) of the channel estimate for the different channel estimation approaches in Fig. 7. Ideal LMMSE assumes perfect knowledge of the covariance matrix of the channel as well as the noise power. The performance of the simple LS filtered solution is close to the performance of the LMMSE assuming a uniform channel $(<1 \mathrm{~dB})$ and significantly outperforms the LS estimate with a gain larger than $5 \mathrm{~dB}$.

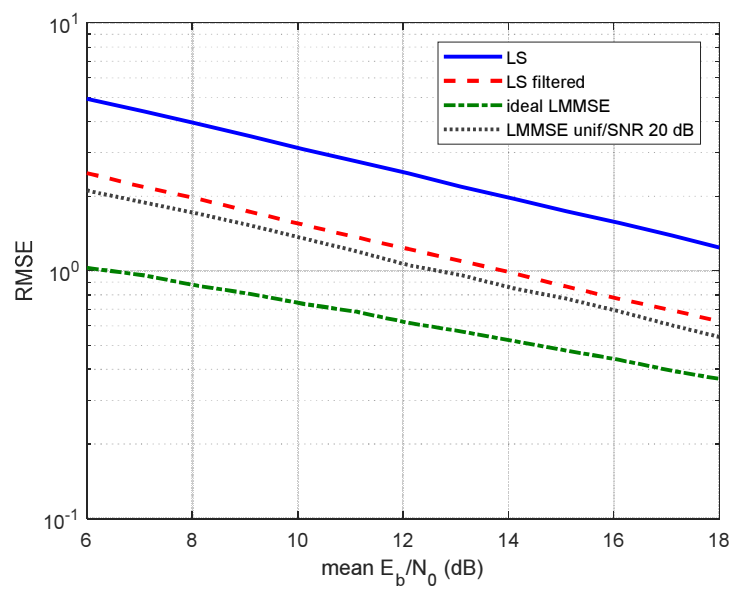

Fig. 7. RMSE of channel estimators in ETU channel (perfect synchronization)

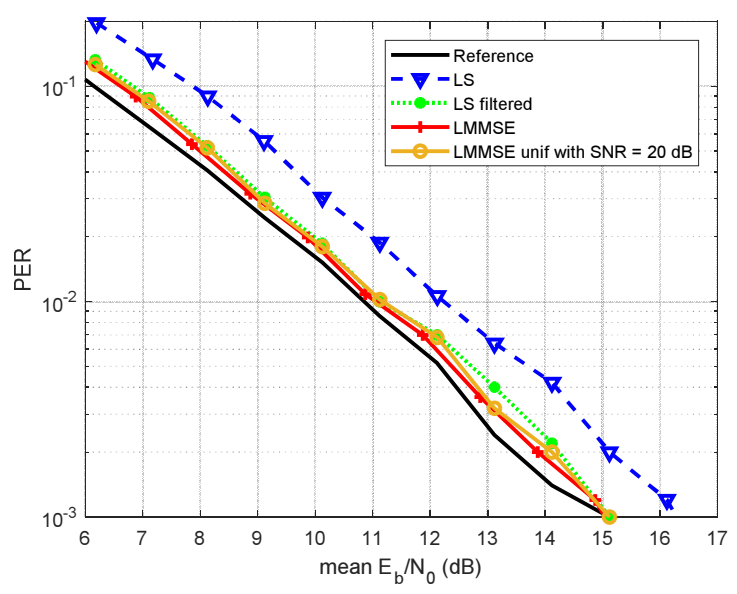

Fig. 8. PER in presence of ETU channel for different channel estimation techniques when perfect synchronization is considered.

In terms of PER, performance is even closer when LS filtered and LMMSE are compared (Fig. 8). For these simulation scenarios, the pilot sequence is repeated 64 times and averaged to improve the performance of the estimator. Except the LS estimator that has a loss of $1.4 \mathrm{~dB}$ in comparison to the reference (perfect channel estimation), the other estimators have a very limited loss between $0.2 \mathrm{~dB}$ (LMMSE) and $0.3 \mathrm{~dB}$ (LMMSE with uniform assumption). Consequently, the LS filtered solution is chosen, as it is a good compromise between complexity and performance.

\section{OVERALl PERFORMANCE OF TURBO-FSK}

The main issue to consider in the overall performance of the Turbo-FSK receiver is the influence of the CFO on the channel estimation. The accuracy of the CFO estimation has been evaluated as not sufficient to consider channel estimation only on a preamble for performance over the entire packet. The probability of a phase rotation greater than $2 \pi$ over the $30 \mathrm{~ms}$ duration of the packet is expected to be significant (greater than $1 \%$ as seen in section II.B).

To address this issue, a second CFO estimation is made on the distributed pilots used for channel estimation also using the Mengali algorithm [12]. Distributed pilot symbols have been regularly spaced over the entire transmission burst. Three different cases have been considered: one pilot symbol every 6 symbols, one pilot symbol every 10 symbols and one pilot symbol every 20 symbols. The preamble is made of $N_{a} \times N_{p}=$ $130 \times 32$ samples. A second CFO correction is done followed by channel estimation on the averaged distributed pilot symbols. Channel and CFO are both assumed constant over the duration of the burst.

For AWGN channel, the loss of $E_{b} / N_{0}$ for PER levels of $1 \%$ is less than $0.2 \mathrm{~dB}$ when one pilot symbol every 6 symbols (Fig. 9 ) is considered. When the loss of energy introduced by the addition of pilot samples is considered, a penalty of $10 \log _{10}(6 / 5)=0.8 \mathrm{~dB}$ should be added to the performance loss giving an overall performance loss inferior to $1 \mathrm{~dB}$ when both synchronization and channel estimation are taken into account. Similarly, when one pilot symbol every 10 symbols is 


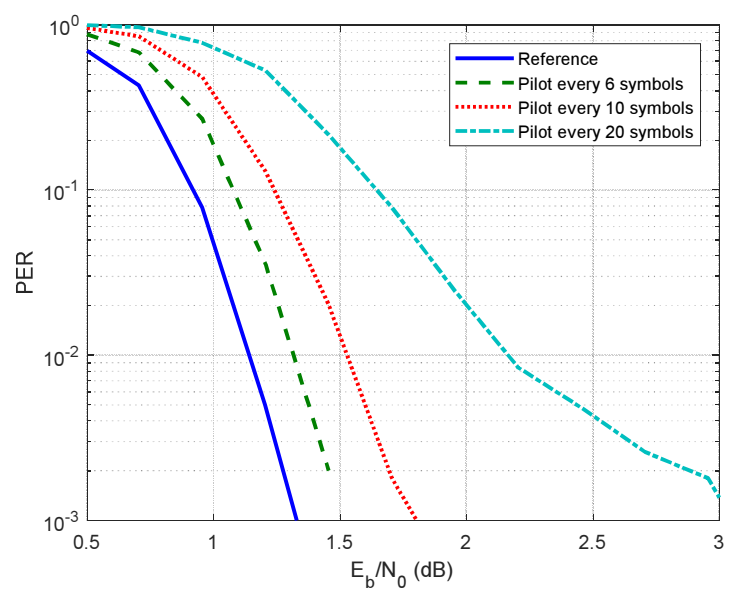

Fig. 9. PER for AWGN channel including synchronization, CFO estimation and correction, channel estimation.

considered, a loss of $0.4+10 \log _{10}(10 / 9)=0.9 \mathrm{~dB}$ is observed: i.e. the same energy per bit has to be transmitted for both cases. When less pilot symbols are considered, the loss is more important ( $>1.4 \mathrm{~dB}$ for one pilot every 20 symbols).

For ETU channel (Fig. 10), similar conclusions can be drawn: the loss of energy efficiency is less than $1.0 \mathrm{~dB}$ when compared to perfect synchronization and channel estimation.

\section{CONCLUSION}

Thanks to its performance very close to Shannon's limit and its constant envelope modulation, Turbo-FSK is a waveform that is well adapted to future LPWA systems notably massive machine type communication applications of the fifth generation of cellular systems. However, the impact of synchronization and channel estimation have so far not been considered on the performance of the Turbo-FSK receiver. This paper demonstrated that using preamble and distributed pilot symbols, performance degradation in required energy per information bit due to imperfect synchronization and channel estimation is fairly limited: less than $1 \mathrm{~dB}$ for AWGN and ETU channels. The

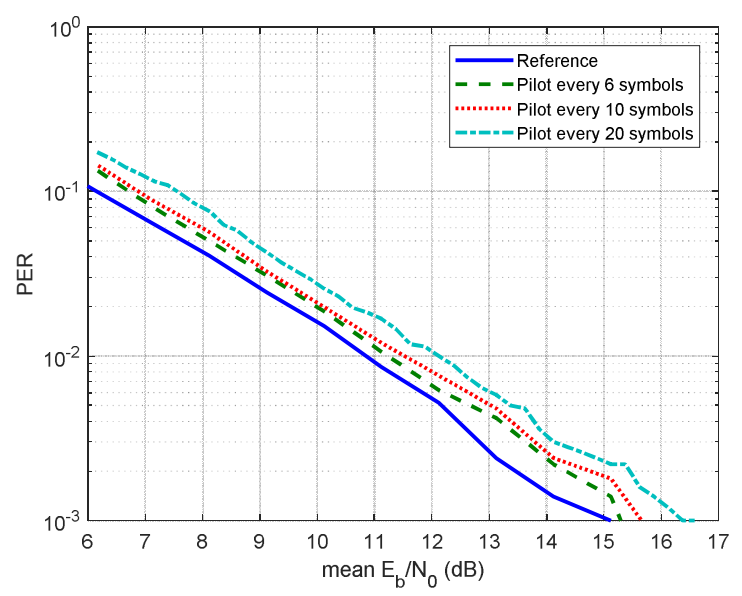

Fig. 10. PER for ETU channel including synchronization, CFO estimation and correction, channel estimation. preamble and the pilots have been specifically designed to have a constant envelope which is of prime importance for energy efficiency at the transmitter. The preamble has also correlation properties such that the use of extra signaling (SFD) is avoided and CFO is tolerated. This approach is thus particularly well adapted to the selected LPWA scenario.

Future work should consider extension of the presented channel estimation scheme to support mobility scenarios.

\section{ACKNOWLEDGMENT}

The research leading to these results was supported by the French Agence Nationale de la Recherche (ANR), under grant agreement ANR-16-CE25-0002 (project EPHYL).

\section{REFERENCES}

[1] Gartner, Inc.: Gartner Says 8.4 Billion Connected "Things" Will Be in Use in 2017, Up 31 Percent From 2016. [Online] https://www.gartner.com/newsroom/id/3598917

[2] U. Raza, P. Kulkarni and M. Sooriyabandara, "Low Power Wide Area Networks: An Overview," in IEEE Communications Surveys \& Tutorials, vol. 19, no. 2, pp. 855-873, Secondquarter 2017.

[3] Berg Insight: Cellular and LPWA IoT Device Ecosystems, 123-134, 4 2017.

[4] Rebbeck, T., Mackenzie, M. Sandell, J. J. van de Beek, S. K. Wilson., Afonso, N.: Low-Powered Wireless Solutions Have the Potential to Increase the M2M Market by Over 3 Billion Connections. Analysys Mason, 2014

[5] Raab, F.H., Asbeck, P., Cripps, S., Kenington, P.B., Popovic, Z.B., Pothecary, N., Sevic, J.F., Sokal, N.O., "Power amplifiers and transmitters for RF and Microwave," IEEE Trans. on Microwave Theory and Techniques 50(3), 814-826, 2002.

[6] Miller, S.L., O'Dea, R.J., "Peak power and bandwidth efficient linear modulation", IEEE Trans. on Communications, vol. 46, no. 12, pp. 1639 1648, Dec 1998

[7] Y. Roth, J.-B. Dore, L. Ros, and V. Berg, "Turbo-FSK: A New Uplink Scheme for Low Power Wide Area Networks," in 2015 IEEE 16th International Workshop on Signal Processing Advances in Wireless Communications (SPAWC), June 2015, pp. 81-85.

[8] Roth, Y.: The Physical Layer for Low Power Wide Area Networks: A Study of Combined Modulation and Coding Associated with an Iterative Receiver. PhD Thesis, Université Grenoble Alpes (July 2017). https://hal.archives-ouvertes.fr/tel-01568794

[9] L. Bahl, J. Cocke, F. Jelinek, and J. Raviv, "Optimal decoding of linear codes for minimizing symbol error rate (corresp.)," IEEE Trans. on Information Theory, vol. 20, no. 2, pp. 284-287, Mar 1974.

[10] D. Chu, "Polyphase codes with good periodic correlation properties", IEEE Trans. Inf. Theory, vol. 18, no. 4, pp. 531-532, 1972.

[11] "3rd Generation Partnership Project (3GPP) Technical Specification Group Radio Access Network; Evolved Universal Terrestrial Radio Access (E-UTRA); Physical channels and modulation (Release 10)", 3GPP TR 36.211, V1O.3.0" Sept. 2011.

[12] U. Mengali and M. Morelli, "Data-aided frequency estimation for burst digital transmission," in IEEE Trans. on Communications, vol. 45, no. 1, pp. 23-25, Jan 1997.

[13] Evolved Universal Terrestrial Radio Access (E-UTRA): Base Station (BS) Radio Transmission and Reception. 3GPP TS 36.104, V14.3.0, Release 14 (2017)

[14] V. Savaux and Y. Louët, "LMMSE channel estimation in OFDM context: a review," in IET Signal Processing, vol. 11

[15] O. Edfors, M. Sandell, J. J. van de Beek, S. K. Wilson and P. O. Borjesson, "OFDM channel estimation by singular value decomposition," in IEEE Trans. on Communications, vol. 46, no. 7, pp. 931-939, Jul 1998 